\title{
Simulating Noisy, Nonparametric, and Multivariate Discrete Patterns
}

by Ruby Sharma, Sajal Kumar, Hua Zhong and Mingzhou Song

\begin{abstract}
Requiring no analytical forms, nonparametric discrete patterns are flexible in representing complex relationships among random variables. This makes them increasingly useful for data-driven applications. However, there appears to be no software tools for simulating nonparametric discrete patterns, which prevents objective evaluation of statistical methods that discover discrete relationships from data. We present a simulator to generate nonparametric discrete functions as contingency tables. User can request strictly many-to-one functional patterns. The simulator can also produce contingency tables representing dependent non-functional and independent relationships. An option is provided to apply random noise to contingency tables. We demonstrate the utility of the simulator by showing the advantage of the FunChisq test over Pearson's chi-square test in detecting functional patterns. This simulator, implemented in the function simulate_tables in the R package FunChisq (version 2.4.0 or greater), offers an important means to evaluate the performance of nonparametric statistical pattern discovery methods.
\end{abstract}

\section{Introduction}

The demand for pattern discovery methodologies has elevated as massive automatic data collection takes place in every application domain. Consequently, an increasingly critical task in data science is to design effective algorithms to recognize complex meaningful patterns from data. Nonparametric discrete patterns do not require an analytical form, allowing them to flexibly represent functional and associative relationships among random variables. This makes them appealing to data-driven capture of complex relationships from big data sources. Indeed, many classical statistical methods can detect associative patterns between discrete random variables, including the widely used Pearson's chisquare test (Pearson, 1900), Fisher's exact test (Fisher, 1922), G-test (McDonald, 2014), and Barnard's test (Barnard, 1945).

However, these methods are not specifically designed for detecting functional patterns, where a dependent variable is a mathematical function of other independent variables. Functional relationships are considered powerful indicators of causality (Simon and Rescher, 1966). Recent methods for detecting discrete functional patterns such as the functional chi-square test (FunChisq) (Zhang and Song, 2013) have demonstrated their effectiveness in identifying causal molecular interactions in human breast cancer from both real and simulated protein abundance data (Hill et al., 2016). To evaluate such methods, software tools that can randomly generate diverse functional patterns are necessary. Such computer programs are unavailable as far as we are aware, in spite of several $\mathrm{R}$ functions for generating other types of random contingency table. The function $r 2 d t a b l e$ in the base package stats creates random two-way tables with given marginals using Patefield's algorithm (Patefield, 1981) under product-multinomial sampling. The package rTableICC (Demirhan, 2016) produces random $2 \times 2 \times K$ tables and $R \times C$ tables based on either intraclass-correlated or uncorrelated individuals. No utility known to us can generate noisy, random, and nonparametric discrete patterns that satisfy requirements on functional relationships between the row and column variables.

To address this gap, we present a new simulator to generate discrete functional patterns. This simulator is publicly available and implemented as the $\mathrm{R}$ function simulate_tables within the $\mathrm{R}$ package FunChisq ( $\geq 2.4 .0$ ) (Zhang et al., 2017), which also contains statistical hypothesis testing methods for non-parametric functional dependencies using asymptotic chi-square or exact distributions. Functional chi-squares are asymmetric and functionally optimal, unique from other related statistics. The simulator can generate associative two-way contingency tables to depict several types of relationships. A relationship type can be a combination of statistical dependency and mathematical functionality. In a dependent relationship, the outcomes of two random variables are statistically dependent; whereas in an independent relationship, the outcomes of two random variables are statistically independent of each other. Dependency can be further categorized as functional (Bartle, 1964) or non-functional. The simulator is also capable of generating many-to-one functional patterns whose inverse is non-functional. To emulate real data often tainted with noise, our simulator can apply a controllable level of house noise (Zhang et al., 2015) into the contingency tables. This option enables one to evaluate the robustness of a discrete pattern discovery algorithm subject to noise. We utilized the simulator to contrast FunChisq and Pearson's chi-square tests and demonstrate their expected difference in detecting discrete functional relationships. We also report the runtime of the simulator as a function of sample size with and without noise. Finally, we illustrated how to use this computer program to generate simulated data for weather forecasting, drug development, molecular biology, 
and statistical algorithm design. This discrete data simulator thus can serve as a benchmarking tool for the development of new statistical methods for pattern discovery in various scientific fields.

\section{Generating random contingency tables with required discrete patterns}

The simulator randomly samples contingency tables with specified functional and statistical patterns and also has an option to apply additional noise on the tables. Let $X \in\{1, \ldots, r\}$ be a discrete random variable with $r$ levels, and $Y \in\{1, \ldots, c\}$ be a second discrete random variable with $c$ levels. We use $p_{X Y}(i, j)$ to represent the joint probability mass function of $X$ and $Y$, and $p_{X}(i)$ and $p_{Y}(j)$ for the marginal probability mass functions of $X$ and $Y$, respectively. We use the notation $Y=f(X)$ to indicate that $Y$ is some function of $X$, and the notation $X \neq g(Y)$ to indicate that $X$ is not any function of $Y$. We use the notation $X \perp Y$ to indicate that $X$ and $Y$ are statistically independent.

We define four pattern types formed by $X$ and $Y$ as follows:

- Functional-Y is a function of $X$;

- Many-to-one- $Y$ is a function of $X$, but not vice versa. A discrete non-monotonic function is not necessarily many-to-one;

- Dependent non-functional $-Y$ and $X$ are statistically dependent, but not a function of each other;

- Independent-Y and $X$ are statistically independent.

For all functional patterns, we do not consider the special case where $X$ or $Y$ can take only one value $(r=1$ or $c=1)$, as in constant functions. We do not include the pattern type where $Y$ is not a function of $X$ but $X$ is a function of $Y$, as it can be generated easily by transposing a many-to-one functional pattern. These four patterns are characterized by the mathematical and statistical relations between $X$ and $Y$ in Table 1.

\begin{tabular}{lccc}
\hline Pattern & Is $Y=f(X) ?$ & Is $X=g(Y) ?$ & Is $X \perp Y ?$ \\
\hline Functional & True & True or False & False \\
Many-to-one & True & False & False \\
Dependent non-functional & False & False & False \\
Independent & False & False & True \\
\hline
\end{tabular}

Table 1: Four pattern types between two random variables $X$ and $Y$. Here functional patterns do not include constant functional patterns.

The simulator will generate three related tables in order: a noise-free sampled contingency table, a pattern table, and a noisy contingency table. All tables are $r \times c$, where $X$ and $Y$ are the row and column variables, respectively. Here is an explanation of these tables:

- The sampled contingency table-With given sample size $n$ and row and/or column marginal probability mass functions, this table satisfies both the functional and statistical requirements. This table, being noise free, can be used to simulate noisy versions and evaluate the performance of pattern discovery algorithms on their statistical effectiveness.

- The binary pattern table-This table is created by setting all non-zero entries in the sampled contingency table to 1 . Thus values in the pattern table are either 0 or 1 . The table strictly satisfies the mathematical relationship for a given pattern type requested by the user, but it does not meet the statistical requirements. It can be used as the ground truth or gold standard for benchmarking how well pattern discovery algorithms can uncover the mathematical relationships.

- The noisy contingency table-At a user-specified noise level, this table is the noisy version of the sampled contingency table. Due to the added noise, this table may no longer strictly satisfy the required functional or statistical relationships. This table is the main output to be used for the evaluation of a discrete pattern discovery algorithm.

Figure 1 illustrates the four pattern types by sampled contingency tables generated using the simulator. The tables are rotated so that the horizontal axis represents the row variable $X$.

Next, we describe how to generate the sampled contingency tables for each type of discrete pattern. All pattern types require the common input of sample size $n$ and table size $r \times c$. They differ in which marginal probability mass functions, $p_{X}, p_{Y}$, or both, must be provided. Let $n_{i j}$ be the count in the 

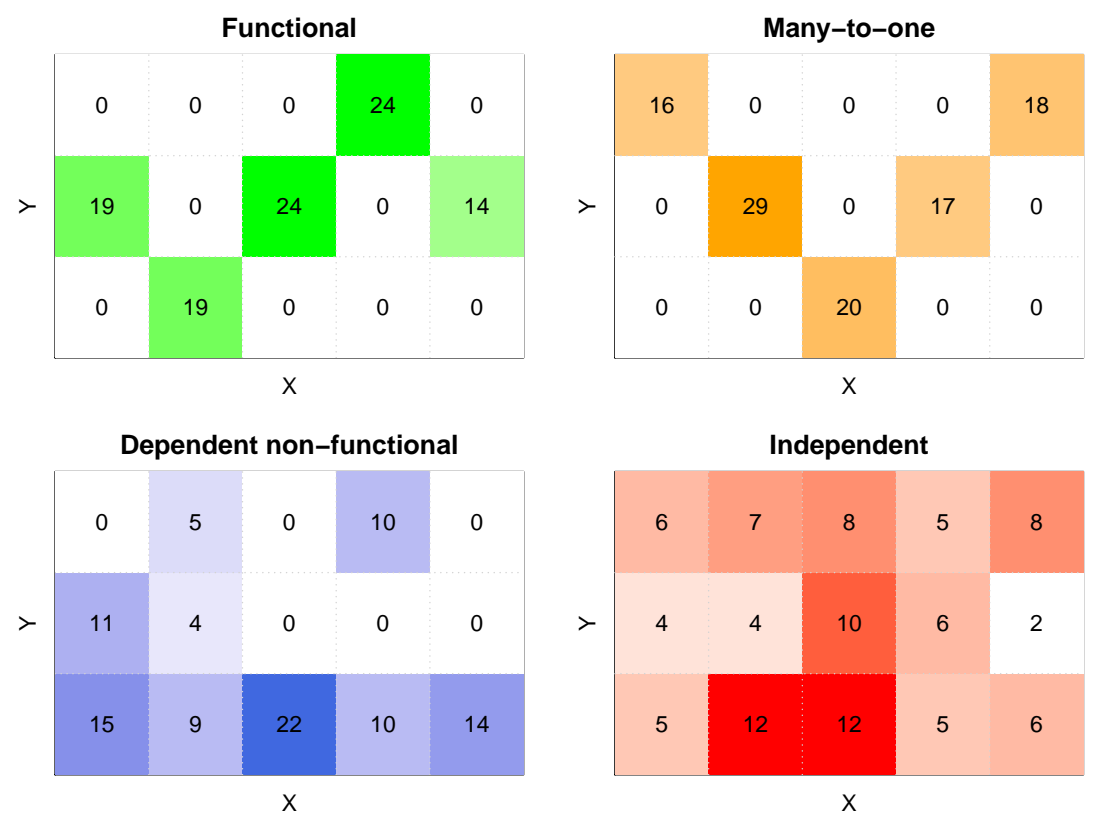

Figure 1: Four pattern types generated by the simulator. The contingency tables are rotated so that row variable $X$ is the horizontal axis. The original tables are all $5 \times 3$ with a sample size of 100 . The number in each square is the count in the corresponding table entry. No noise is applied. The color intensity of each entry is proportional to the sample count in that entry.

entry at row $i$ and column $j$. We use the notation $n_{i}$. to indicate the sum of row $i$ in a contingency table:

$$
n_{i .}=\sum_{j=1}^{c} n_{i j}, \quad i=1, \ldots, r
$$

and $n_{\cdot j}$ as the sum of column $j$ :

$$
n_{\cdot j}=\sum_{i=1}^{r} n_{i j}, \quad j=1, \ldots, c
$$

\section{Generating functional tables}

Functional patterns can be used to model causal relationships that are either linear or nonlinear. A contingency table reduces the burden of assuming a parametric form for the function. In a functional table representing $Y=f(X)$, every level $X=i$ maps to exactly one outcome $Y=j$ in the contingency table. The following steps generate a noise-free sampled table that satisfies all mathematical and statistical requirements:

Input: row marginal probability function $p_{X}$, sample size $n \geq 2$, table size $r \times c, r, c \geq 2$.

Output: a non-constant functional table.

1. Randomly generate the row sums $n_{i}$. $(i=1, \ldots, r)$ by the multinomial distribution with success probability function $p_{X}$ and the sample size $n$.

2. For each row $i$ in the table, initialize the entire row to be 0 , randomly pick a column $j \in\{1, \ldots, c\}$ with equal probability, and set $n_{i j}=n_{i}$.

3. Convert the function from constant to non-constant: If the function is constant-all nonzero values are on the same column $j$, randomly pick a row $i^{\prime}$ from $\{1, \ldots, r\}$ with equal probability and a column $j^{\prime}$ from $\{1, \ldots, c\} \backslash\{j\}$ with equal probability. Swap the values of $n_{i j}$ and $n_{i^{\prime} j^{\prime}}$. This will guarantee function $Y=f(X)$ is a non-constant function.

\section{Generating many-to-one functional tables}

Many-to-one functions are special cases of functional relationships. They are increasingly relevant as they can expose complex patterns from data of large sample sizes. Being able to generate such patterns will facilitate the evaluation of complex pattern discovery methods. The following two main steps generate this type of patterns: 
Input: row marginal probability function $p_{X}$, sample size $n \geq 2$, table size $r \times c, r \geq 3, c \geq 2$.

Output: a strictly many-to-one and non-constant functional table.

1. Generate a non-constant functional table using Step 1 to 3 above.

2. Convert the function from one-to-one to many-to-one: If the function is one-to-one-every column of the table has at most one non-zero entry, do the following. Randomly pick a row $i$ from all those rows with non-zero totals with equal probability. Let $j$ be the index to the only non-zero entry on row $i$. Pick $j^{\prime}$ from the indices of those columns with non-zero totals except $j$ with equal probability. Swap the values of $n_{i j}$ and $n_{i j^{\prime}}$.

\section{Generating dependent and non-functional tables}

In non-functional dependent relationships between $X$ and $Y, X$ and $Y$ are statistically dependent but $Y$ is not a function of $X$ and $X$ is neither a function of $Y$. They are not as strong as functional patterns in revealing causal relationships, but can reveal strong associative relationships such as a circular pattern. Their joint probability function must satisfy

$$
p_{X Y}(i, j) \neq p_{X}(i) \cdot p_{Y}(j) \quad \text { for some } i, j
$$

As we do not have the above true probability functions, we will directly estimate them using frequencies in the table. If the above inequality is satisfied, we call $X$ and $Y$ empirically statistically dependent; otherwise, $X$ and $Y$ are empirically statistically independent.

Lemma 1. Let $p, P, Q, M, N$ be five positive numbers and $q$ be a nonnegative number, where $P>p$ and $Q>q$. If these numbers satisfy two equalities

$$
\frac{P}{N} \cdot \frac{M}{N}=\frac{p}{N}
$$

and

$$
\frac{Q}{N} \cdot \frac{M}{N}=\frac{q}{N}
$$

and if we are given a number $k$ such that $0<k \leq p$, then the following two inequalities must be true:

$$
\frac{P-k}{N} \cdot \frac{M}{N}>\frac{p-k}{N}
$$

and

$$
\frac{Q+k}{N} \cdot \frac{M}{N}<\frac{q+k}{N}
$$

Proof. As we are given $P>p$, we have algebraically

$$
\frac{P-k}{p-k}=\frac{P-p}{p-k}+1
$$

monotonically increasing as $k$ increases from 0 to $p$. Thus it follows

$$
\frac{P-k}{p-k}>\frac{P}{p}=\frac{N}{M}
$$

using the equality in Eq. (4). Multiplying both sides by $\frac{(p-k) M}{N^{2}}$, we immediately prove inequality (6). Similarly, with given $Q>q$ and as $k$ increases from 0 , we have a monotonic decreasing function of $k$

$$
\frac{Q+k}{q+k}=\frac{Q-q}{q+k}+1
$$

which implies

$$
\frac{Q+k}{q+k}<\frac{Q}{q}=\frac{N}{M}
$$

based on Eq. (5). Multiplying both sides by $\frac{(q+k) M}{N^{2}}$, we immediately prove inequality (7).

Based on Lemma 1, we design Algorithm 1 to break the statistical independency. Let $(i, j)$ represent the entry at row $i$ and column $j$.

Algorithm 1. Convert an independent non-functional table to a dependent non-functional table.

Input: an independent non-functional table

Output: a dependent non-functional table 
1. Randomly pick an entry $(i, j)$ with at least one sample point $\left(n_{i j}>0\right)$.

2. Randomly select column $j^{\prime} \neq j$ with equal probability from row $i$.

3. Move all $n_{i j}$ samples from entry $(i, j)$ to $\left(i, j^{\prime}\right)$, which finally have 0 and $n_{i j}+n_{i j^{\prime}}$ sample points, respectively.

Theorem 1. An empirically statistically independent non-functional contingency table can be converted to dependent non-functional contingency table by Algorithm 1. The row marginal probability function remains the same after the conversion.

Proof. Table 2 illustrates that any independent non-functional table has at least two rows and two columns with non-zero entries. Non-functionality guarantees at least one row (row $i_{1}$ in Table 2 ) and one column (column $j_{1}$ in Table 2) each with two non-zero entries. The independent property ensures that at least another non-zero row (row $i_{2}$ in Table 2) and another non-zero column (column $j_{2}$ in Table 2) has the same distribution of numbers proportional to row $i_{1}$ and the column $j_{1}$, respectively. Additionally, all non-zero rows are proportional to each other by a constant, and so do the columns.

\begin{tabular}{|c|c|c|c|c|}
\hline$\cdots$ & $\cdots$ & $\cdots$ & $\cdots$ & $\cdots$ \\
\hline$\cdots$ & $n_{i_{1} j_{1}}>0$ & $\cdots$ & $n_{i_{1} j_{2}}>0$ & $\cdots$ \\
\hline$\cdots$ & $\cdots$ & $\cdots$ & $\cdots$ & $\cdots$ \\
\hline$\cdots$ & $n_{i_{2} j_{1}}>0$ & $\cdots$ & $n_{i_{2} j_{2}}>0$ & $\cdots$ \\
\hline$\cdots$ & $\cdots$ & $\cdots$ & $\cdots$ & $\cdots$ \\
\hline
\end{tabular}

Table 2: Any independent non-functional table has at least two rows and two columns each with at least two non-zero entries.

In the input table to Algorithm 1, entry $(i, j)$ satisfies the empirical independence equation

$$
\frac{n_{i} \cdot}{n} \cdot \frac{n_{\cdot j}}{n}=\frac{n_{i j}}{n}
$$

where $n_{i}$. is the sum of row $i$ and $n_{. j}$ is the sum of column $j$. After the move, we have by Lemma 1

$$
\frac{n_{i \cdot} \cdot n_{\cdot j}-n_{i j}}{n}>\frac{0}{n}
$$

where $n_{. j}-n_{i j}>0$ is guaranteed by the property shown in Table 2. As the left hand side of the inequality is the product of the new marginal probabilities and the right hand side is the new joint probability, it implies the loss of statistical independency in the modified table. Therefore the modified table is now statistically dependent.

Also by the property shown in Table 2, no matter where $n_{i j}$ was moved to, we will indeed have another row and another column with at least two non-zero entries, which will guarantee the non-functionality of the modified table.

As the samples are moved within the same row $i$, the row sum is unchanged and thus the row marginal probability function is unchanged in the modified table.

Therefore, we conclude that the new table must be dependent non-functional.

Such tables are only possible when $n \geq 4$. The following steps generate such tables by distributing samples to rows and then to columns and converting any resulting functions to non-functions:

Input: row marginal probability function $p_{X}$, sample size $n \geq 4$, table size $r \times c, r \geq 2$, and $c \geq 2$.

Output: a dependent non-functional table.

1. Randomly generate the row sums $n_{i}$. $(i=1, \ldots, r)$ by the multinomial distribution with success probability function $p_{X}$ and the sample size $n$.

2. For each row $i$ in the table, initialize the entire row to be 0 . Sample a value $k$ from $\{1, \ldots, c\}$ with equal probability. Randomly pick $k$ columns $j_{1}, \ldots, j_{k}$ from $\{1, \ldots, c\}$ with equal probability. Then randomly determine the values of $n_{i j_{1}}, \ldots, n_{i j_{k}}$ using the multinomial distribution with equal success probability of $1 / k$.

3. If $Y=f(X)$, convert the function to a non-function: If the table represents a valid functioneach row has at most one non-zero entry, do the following. Pick a row $i$ from all rows with at least two samples with equal probability. Let $j$ be the index to the only non-zero entry in row $i$. Pick $j^{\prime}$ from $\{1, \ldots, c\} \backslash\{j\}$ with equal probability. Redistribute the row sample $n_{i}$. into $n_{i j}$ and $n_{i j^{\prime}}$ with equal probability using a binomial distribution. 
4. If $X=g(Y)$, convert the function to a non-function: follow the previous step but switch rows and columns. This operation also maintains the non-functionality $Y \neq f(X)$ as no non-zero entries are eliminated.

5. If empirically $X \perp Y$, we break the statistical independency by converting the table to a dependent non-functional table using Algorithm 1.

\section{Generating independent tables}

When $X$ and $Y$ are statistically independent, their joint probability mass function is

$$
p_{X Y}(i, j)=p_{X}(i) \cdot p_{Y}(j) \quad \text { for all } i \in\{1, \ldots, r\} \text { and } j \in\{1, \ldots, c\}
$$

where $p_{X}(i)$ and $p_{Y}(j)$ are marginal probabilities of occurrence for $X=i$ and $Y=j$, respectively. The tables formed by such $X$ and $Y$ are useful as a negative control, because they represent patterns that are typically considered uninteresting. To generate an independent pattern, the input needs both row and column marginal probability mass functions $p_{X}$ and $p_{Y}$. As $X$ and $Y$ are statistically independent, the numbers of samples in each entry follow a multinomial distribution. The success probability in the entry at row $i$ and column $j$ is exactly $p_{X Y}(i, j)$. Given the sample size $n$, we randomly generate samples from the multinomial distribution with $r \times c$ outcomes and map them to the sampled contingency table.

\section{The house noise model}

It is very common to observe noise in real data; noise may arise due to a multitude of reasons ranging from data preparation through the machinery involved in the entire data acquisition process. In statistical inference, noise needs to be handled to reduce type I and type II errors. Thus, our simulator also factors in noise to make the contingency tables resemble those constructed out of real data sets in order to additionally provide for a test of robustness.

By specifying the noise level parameter in the simulate_tables function, one can apply noise to a contingency table. We use the discrete house noise model (Zhang et al., 2015) that is controlled by the noise level parameter $\theta . \theta$ is a continuous constant between 0 and 1 , where 0 represents no noise and 1 represents maximum noise level. Let $Y \in\{1, \ldots, c\}$ be a dependent discrete random variable and $c>1$ be the number of discrete levels of $Y$. Let $Y^{\prime} \in\{1, \ldots, c\}$ be a random discrete variable which represents the noisy version of $Y$. Let $y$ and $y^{\prime}$ represent values for $Y$ and $Y^{\prime}$, respectively. The house noise model is defined through a probability function of noisy $Y^{\prime}$ conditioned on truth $Y$ and noise level $\theta$ by

$$
p_{Y^{\prime} \mid Y}\left(y^{\prime} \mid y, \theta\right)=\operatorname{Pr}\left(Y^{\prime}=y^{\prime} \mid Y=y, \theta\right)= \begin{cases}{\left[\left(1-\frac{\left|y-y^{\prime}\right|}{\sum_{d=1}^{c}(|d-y|)}\right) \frac{\theta}{c-1}\right](1-\theta)+\frac{\theta}{c}} & \text { if } y^{\prime} \neq y \\ \left(\frac{\theta}{c-1}+1-\theta\right)(1-\theta)+\frac{\theta}{c} & \text { if } y^{\prime}=y\end{cases}
$$

The model works on the principle of making its value closer to the true value rather than being further away. By this model, the probability of a larger deviation of $Y^{\prime}$ from $Y$ is smaller than that of a smaller deviation. At $\theta=0$, we have $Y^{\prime}=Y$ and it is thus noise free; at $\theta=1$, the conditional probability function becomes uniformly distributed and the noise completely destroys the underlying patterns.

We implemented the model as an $\mathrm{R}$ function add. house. noise to apply noise to an input contingency table and return a noisy contingency table as the output. The syntax of the function is as follows:

add. house. noise(tables, $u$, margin $=1$ )

where tables is a list of input contingency tables and $u$ is the noise level between 0 and 1 . The noise can be applied along rows (margin=1), columns (margin=2), or both rows and columns (margin=0). In simulate_tables, the noise is always applied along the rows, which can be interpreted as applying noise to the dependent variable $Y$ if the column variable is a function of the row variable $X$. The house noise model can be used independently of function simulate_tables.

\section{Performance evaluation}

To affirm each table type generated by the simulator indeed has the required characteristics, we compared two hypothesis testing methods: the fun.chisq. test (Zhang and Song, 2013) in the R package FunChisq and chisq. test in the R package stats. 
We first simulated 1000 randomly generated tables for each of the four types at the noise level of 0.1 . The numbers of rows and columns of the tables were randomly selected from 3 to 10 . The sample size of each table was randomly drawn from 10 to 1000 and must be at least the table size.

Next we applied the two tests on the tables.The $\log p$-value distributions of both tests are shown in Fig. 2. In Fig. 2a, the FunChisq test tends to yield smaller $p$-values compared to Pearson's chi-square on functional patterns. In Fig. $2 b$, both tests are approximately equal on dependent non-functional patterns. The $p$-values remarkably increased in comparison to functional patterns but are still showing significance due to statistical dependency in the pattern. Taken together, the FunChisq test has a higher sensitivity of functional over non-functional patterns than Pearson's chi-square test, as expected.

In Fig. 2c, the FunChisq test was performed first on noisy many-to-one functional patterns and second on the transposed table. There is an increase in the $p$-value by the latter which explains that the transposed noisy many-to-one tables are no longer functional. We did not show Pearson's chi-square $p$-values because they are equal on transposed contingency tables. Indeed, one expects to see such a difference in the distribution of FunChisq statistics when comparing functional tables versus their transposed non-functional tables, as FunChisq, unlike Pearson's chi squares, is asymmetric when testing functional dependency.

In Fig. $2 \mathrm{~d}$ the $\log p$-value distributions of both FunChisq and Pearson's chi-squares are nearly identical and the $p$-values are very close to 1 , because both tests follow the same $\chi^{2}$ distribution under the null hypothesis when using independent patterns.

These results confirm that our simulator was indeed able to generate functional, non-functional, many-to-one, and independent patterns.

We further benchmarked the runtime of the simulator over four table types, four sample sizes (100, $500,1000$, and 10000), and two noise levels ( 0 and 0.5$)$ at a fixed table size of $5 \times 5$. This gives a total of 32 configurations. For each configuration, we repeated the simulation 100 times to account for random variations. We ran the simulation on iMac (27-inch, Mid 2010) with $2.93 \mathrm{GHz}$ Intel Core i7 processor and 16 GB 1333 MHz DDR3 RAM. Figure 3 shows the runtime of generating noise-free and noisy versions of all four pattern types. It is apparent from the figure that table type, sample size, and noise application can all influence the runtime of table generation. Dependent non-functional tables took the most time because they are subject to more mathematical requirements. Applying noise incurred additional time for all table types. Sample size has an observable effect on the runtime when noise is applied.

\section{Installation and examples of discrete pattern generation}

Here we demonstrate the usage of the simulator by providing examples of all four pattern types including the description of important parameters. The R package FunChisq $(\geq 2.4 .0)$ contains the function simulate_tables that implements the simulator. The package is publicly available from the Comprehensive R Archive Network (CRAN). The package can be installed and loaded by

install. packages ("FunChisq")

library ("FunChisq")

The signature of the function simulate_tables is given below:

simulate_tables $(n=100$, nrow=3, ncol=3, type=c("functional", "many.to. one", "independent", "dependent.non. functional"), noise $=0.0$, n. tables $=1$, row.marginal=rep(1/nrow, nrow), col.marginal=rep(1/ncol, ncol))

The arguments are

- $\mathrm{n}$-an integer specifying the sample size to be distributed in the table. For "functional" and "many. to. one" tables, $\mathrm{n}$ must be no less than nrow. For "independent" tables, $\mathrm{n}$ must be no less than nrow*ncol. For "dependent. non. functional" tables, n must be greater than nrow.

- nrow-an integer specifying the number of rows of output tables. The value must be no less than 2. For "many. to. one" tables, nrow must be no less than 3.

- ncol-an integer value specifying the number of columns in desired table. The value for ncol must be no less than 2 .

- type-a character string to specify the type of pattern underlying the table. The options are "functional" (default), "many.to. one", "independent", and "dependent.non. functional".

- noise-a numeric value between 0 and 1 specifying the factor of noise to be added to the table using the house noise model (Zhang et al., 2015). The house noise is applied along the rows of the table. See add. house. noise for details. 
a

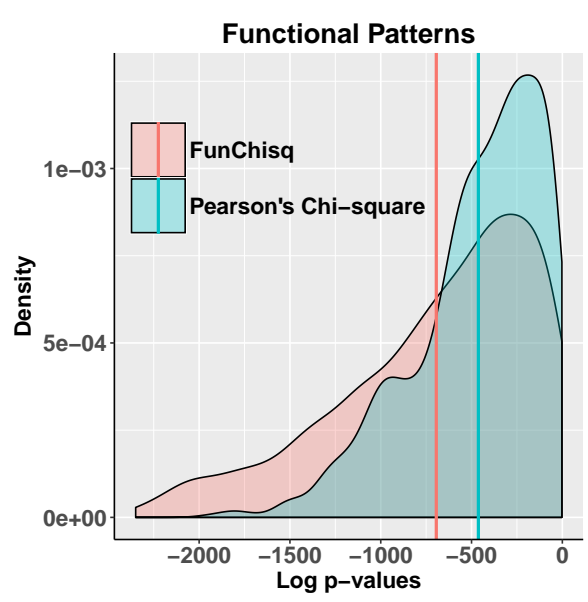

C

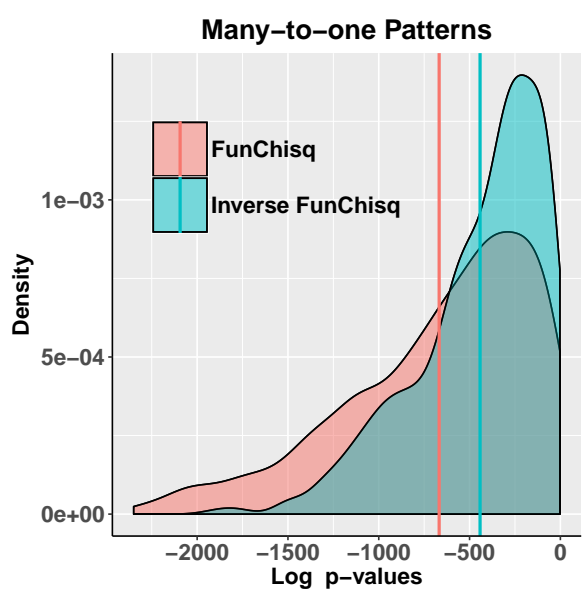

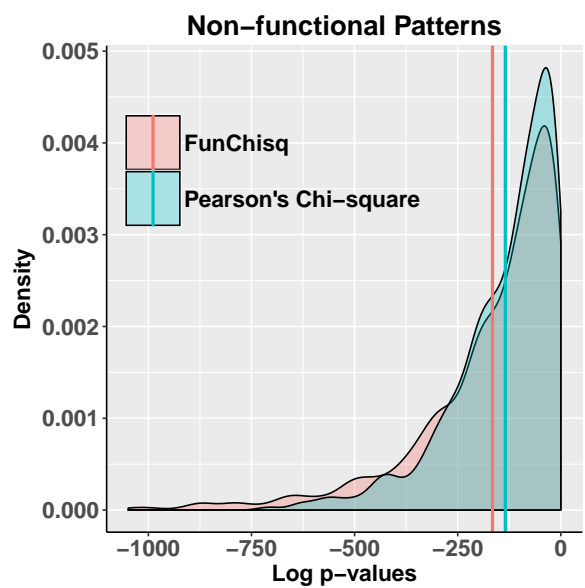

d

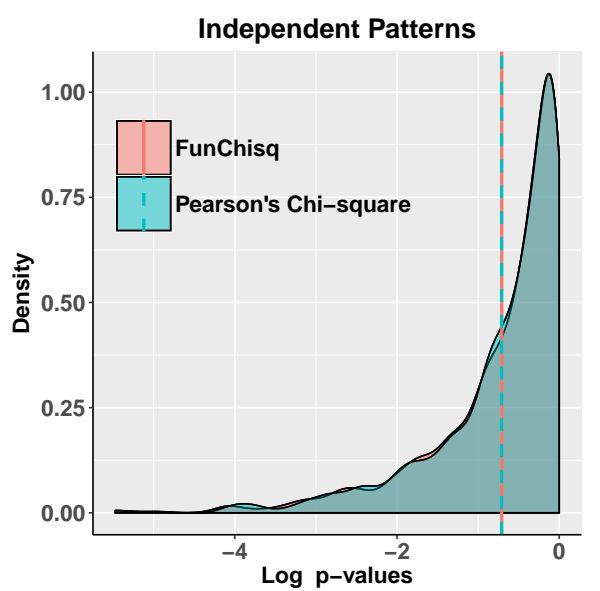

Figure 2: The FunChisq test is more sensitive to functional patterns than Pearson's chi-square test, while being equally sensitive to non-functional patterns. The distributions of $\log p$-values for all four table types using the two tests at a noise level of 0.1 are shown. The means of the distributions are indicated by the vertical lines. (a) Noisy functional patterns. (b) Noisy dependent non-functional patterns. (c) Noisy many-to-one functional patterns. The FunChisq test was applied on both the original table and the transposed table, indicated by inverse FunChisq. (d) Noisy independent patterns.

- n. tables-an integer value specifying the number of tables to be generated.

- row.marginal-a numeric vector of length at least 2 specifying row marginal probabilities. For "many. to. one" tables, the length of row.marginal vector must be no less than 3.

- col.marginal-a numeric vector specifying column marginal probabilities. It is only applicable in generating independent tables.

The return value of the function simulate_tables is a list containing the following components:

- pattern. list-a list of tables containing 0-1 binary patterns. Each table is created by setting all non-zero entries in the corresponding sampled contingency table from sample. list to 1 . Each table strictly satisfies the functional relationship for a given pattern type requested. This table does not meet the statistical requirements. As each table represents the truth regarding the mathematical relationship between the row and column variables, they can be used as the ground truth or gold standard for benchmarking.

- sample. list-a list of tables satisfying both the functional and statistical requirements. These tables are noise free.

- noise. list-a list of tables after applying noise to the corresponding tables in sample. list. Each table is the noisy version of the sampled contingency table. Due to the added noise, each table may no longer strictly satisfy the required functional or statistical relationships. These tables are the main output to be used for the evaluation of a discrete pattern discovery algorithm. 
a

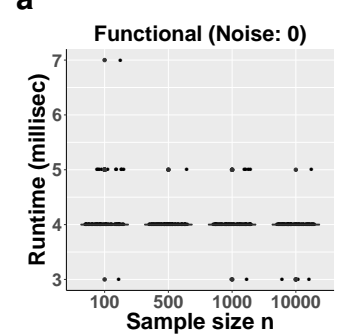

b

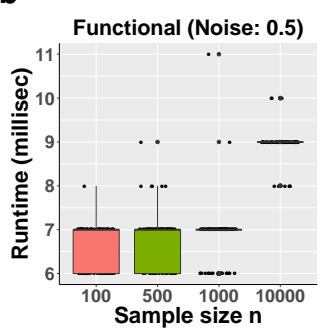

C

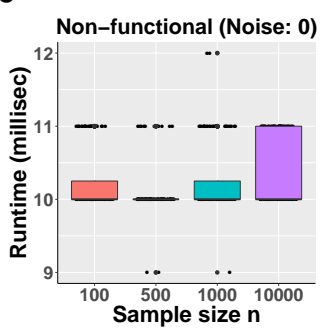

d

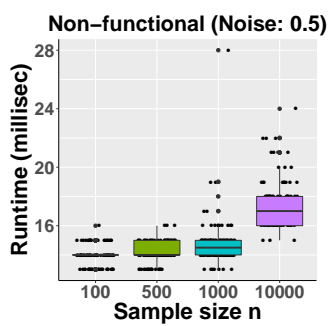

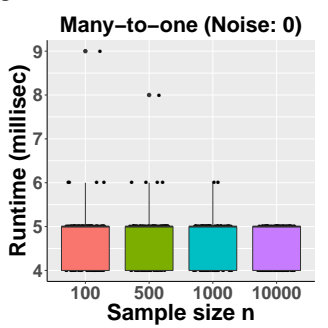

f

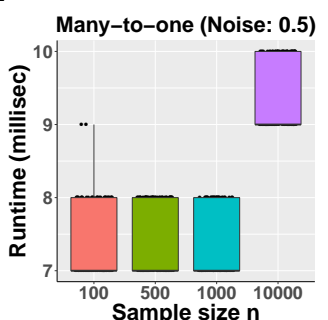

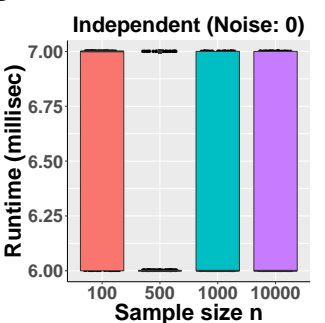

h

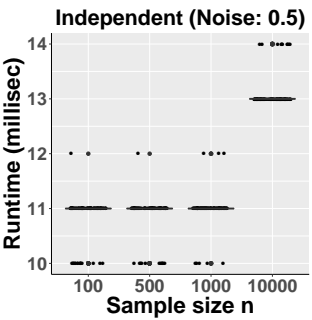

Figure 3: The empirical runtime of generating a contingency table as a function of sample size over four table types and two noise levels. We fix the table size to be $5 \times 5$. The runtime is measured in milliseconds and the box plots are colored by sample sizes. The runtime differs in sample size, table type, and whether noise was applied, as shown in (a) noise-free and (b) noisy functional patterns, (c) noise-free and (d) noisy dependent non-functional patterns, (e) noise-free and (f) noisy many-to-one functional patterns, and $(\mathrm{g})$ noise-free and $(\mathrm{h})$ noisy independent patterns.

- pvalue. list-a list of $p$-values reporting the statistical significance of the generated tables for the required type. When the pattern type specifies a functional relationship, the $p$-values are computed by the FunChisq test; otherwise, the Pearson's chi-square test of independence is used to calculate the $p$-value.

Example 1. A functional table. A scientist working at a weather forecasting agency came up with a novel method that can be trained for each geographical area to predict the amount of rainfall in that area given a month. While the agency is busy collecting real data for the last 10 years, the scientist wants to test his method and train the method to learn the following hypothesis: 'The amount of rainfall in an area is a function of time, expressed in months'. Using different probability distributions for each month (row.marginal) the scientist can simulate contingency tables with $X$ being month with 12 levels and $Y$ being the amount of rainfall with 3 levels 'Scant', 'Medium' and 'Heavy'. We assume that the amount of rainfall is equally likely in a given month. Noise can be added to imitate imperfect real-world scenario. The following code generates $12 \times 3$ functional contingency tables with 100 samples using multinomial distribution at the noise level of of 0.1 :

\begin{tabular}{|c|c|c|c|c|c|c|c|c|c|c|c|}
\hline \multirow{2}{*}{\multicolumn{4}{|c|}{$\begin{array}{l}\text { Output: } \\
\text { pattern.table } \\
\text { C1 C2 C3 }\end{array}$}} & \multicolumn{4}{|c|}{ sampled.table } & \multicolumn{4}{|c|}{ noise.table } \\
\hline & & & & & $\mathrm{C} 1$ & $\mathrm{C} 2$ & C3 & & $\mathrm{C} 1$ & $\mathrm{C} 2$ & C3 \\
\hline R1 & 0 & 1 & 0 & R1 & 0 & 5 & 0 & R1 & 0 & 5 & 0 \\
\hline R2 & 0 & 0 & 1 & R2 & 0 & 0 & 17 & R2 & 0 & 1 & 16 \\
\hline R3 & 0 & 0 & 1 & R3 & 0 & 0 & 3 & R3 & 0 & 0 & 3 \\
\hline R4 & 0 & 1 & 0 & R4 & 0 & 15 & 0 & R4 & 0 & 14 & 1 \\
\hline R5 & 0 & 1 & 0 & R5 & 0 & 3 & 0 & R5 & 1 & 2 & 0 \\
\hline R6 & 0 & 0 & 1 & R6 & 0 & 0 & 5 & R6 & 1 & 0 & 4 \\
\hline R7 & 1 & 0 & 0 & R7 & 22 & 0 & 0 & R7 & 20 & 1 & 1 \\
\hline R8 & 0 & 1 & 0 & $\mathrm{R} 8$ & 0 & 15 & 0 & R8 & 1 & 13 & 1 \\
\hline R9 & 0 & 0 & 1 & R9 & 0 & 0 & 4 & R9 & 0 & 1 & 3 \\
\hline R10 & 1 & 0 & 0 & $\mathrm{R} 10$ & 5 & 0 & 0 & $\mathrm{R} 10$ & 5 & 0 & 0 \\
\hline R11 & 0 & 0 & 1 & R11 & 0 & 0 & 3 & R11 & 0 & 0 & 3 \\
\hline R12 & 0 & 0 & 1 & $\mathrm{R} 12$ & 0 & 0 & 3 & $\mathrm{R} 12$ & 0 & 0 & 3 \\
\hline
\end{tabular}

The scientist can also tune parameters and generate 100 or more such tables representing data for 100 or more years. 
Example 2. A many-to-one functional table. This type of table is a special case of functional table. It can be used to model the relationship between the effectiveness $Y$ and the dosage $X$ of a drug. When the dosage of the drug is too low or too high, it becomes ineffective. The following code generates $4 \times 5$, noisy, many-to-one contingency tables with 100 samples distributed using multinomial distribution. The row marginals used are $0.1,0.3,0.2,0.4$. Noise factor of 0.5 is added to the sampled table.

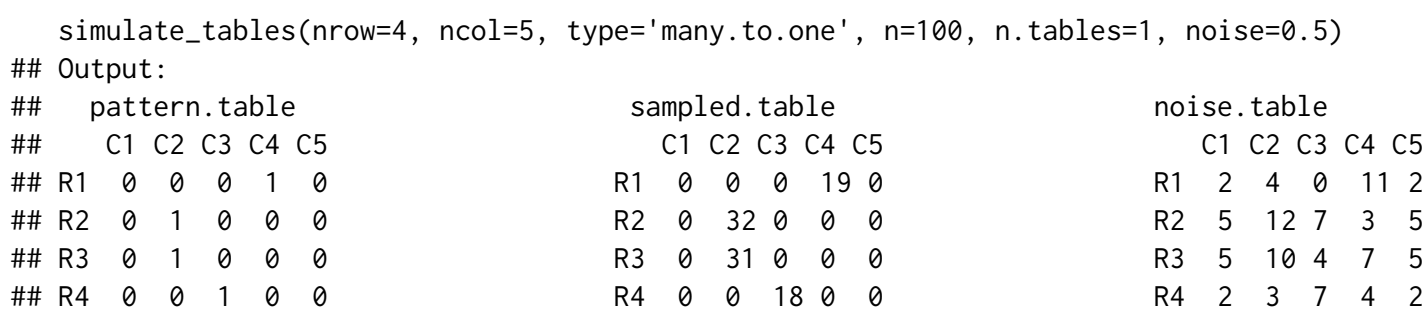

Example 3. An independent table. This type of table is useful in generating null distribution or bootstrapping. To measure the statistical significance or probability of getting a certain empirical score reported by a method, one needs to randomly sample from an independent and identically distributed population. The following code generates $4 \times 5$ contingency tables with 100 samples distributed using multinomial distribution, where row and column variables are statistically independent. The row marginal probabilities are $0.1,0.3,0.2,0.4$ and column marginal probabilities are $0.3,0.2,0.1,0.3,0.1$. No noise is applied.

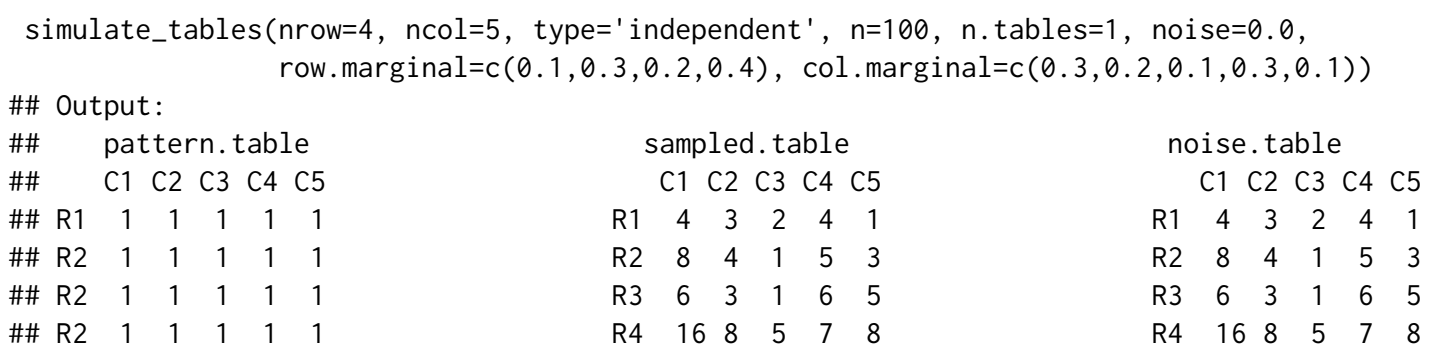

Example 4. A dependent non-functional table. Two genes $X$ and $Y$ may be regulated by a common transcription factor. Here $X$ and $Y$ are categorized as low, medium and high. The two variables are dependent but not a function of each other. We simulate this type of data using dependent non-functional table type. The following code generates $3 \times 3$, noise free, non-functional contingency tables with 100 samples distributed using multinomial distribution. The row marginals used are 0.3 , $0.5,0.2$.

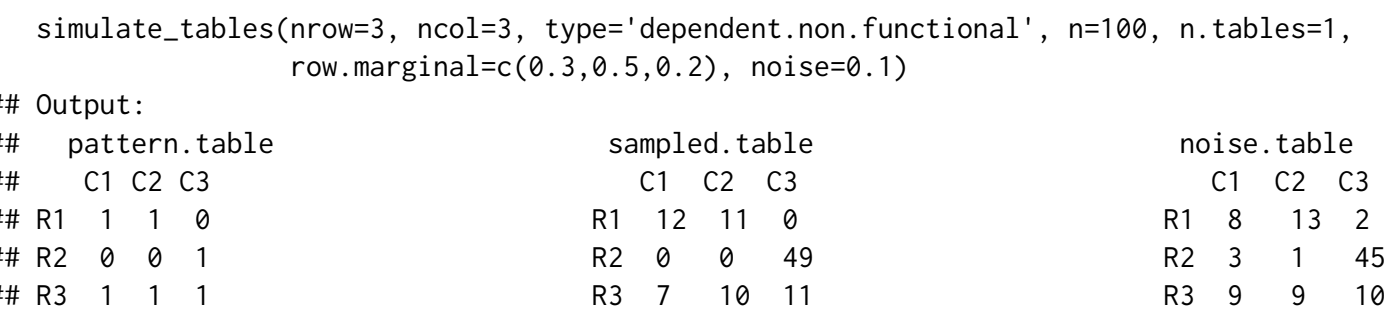

\section{Discussion}

We have just introduced a new simulator which conveniently generates random noisy $r \times c$ contingency tables exhibiting four contrasting pattern types including functional, many-to-one functional, dependent non-functional, and independent. These contingency tables are advantageous for methods which are designed to discover patterns among discrete random variables.

Although other methods for constructing random contingency tables are available (Demirhan, 2016), the generation of functional tables is innovative. Having diverse functional tables meets a need to detect causal relationships from functional dependencies without using a parametric form.

For practical reasons, if the row marginal is non-zero, we will generate at least one sample for that row. Currently the simulator utilizes row marginal probabilities for the generation of dependent contingency tables; in the future we may provide an option to use the column marginal probabilities instead, to match some experimental design where the distribution of the effect variable is predefined, such as in case-control studies of cancer. 
The generated dependent non-functional patterns may span a wide range of statistical dependency from being very weak to very strong. The $p$-values associated with these patterns can be used to filter out tables of weak statistical dependency.

Beyond generating tables of bivariate patterns between the row and column variables, one may consider the row variable as a combination of multiple discrete variables. Therefore one can immediately extend the procedures to generate contingency tables representing multivariate patterns.

In summary, we described algorithms and implemented a simulator to construct contingency tables of desired mathematical and statistical properties, and illustrated the use of this function with several examples. We validated the generation of all table types by the FunChisq and Pearson's chi-square tests. We evaluated the runtime of the function in generating various noisy patterns. This function offers a previously overlooked utility to generate diverse functional patterns to evaluate discrete pattern discovery methods increasingly important in data science research.

\section{Acknowledgment}

This work is supported by US NSF Advances in Biological Informatics Grant DBI 1661331.

\section{Bibliography}

G. A. Barnard. A new test for $2 \times 2$ tables. Nature, 156:177, 1945. URL https://doi.org/10.1038/ 156177a0. [p366]

R. G. Bartle. The Elements of Real Analysis, volume 2. John Wiley \& Sons, 1964. [p366]

H. Demirhan. rTableICC: An R package for random generation of $2 \times 2 \times \mathrm{K}$ and $\mathrm{R} \times \mathrm{C}$ contingency tables. The R Journal, 8(1):48-63, 2016. [p366, 375]

R. A. Fisher. On the interpretation of $\chi^{2}$ from contingency tables, and the calculation of $P$. Journal of the Royal Statistical Society, 85(1):87-94, 1922. URL https://doi .org/10.2307/2340521. [p366]

S. M. Hill, L. M. Heiser, T. Cokelaer, M. Unger, N. K. Nesser, D. E. Carlin, Y. Zhang, A. Sokolov, E. O. Paull, C. K. Wong, K. Graim, A. Bivol, H. Wang, F. Zhu, B. Afsari, L. V. Danilova, A. V. Favorov, W. S. Lee, D. Taylor, C. W. Hu, B. L. Long, D. P. Noren, A. J. Bisberg, The HPN-DREAM Consortium, G. B. Mills, J. W. Gray, M. Kellen, T. Norman, S. Friend, A. A. Qutub, E. J. Fertig, Y. Guan, M. Song, J. M. Stuart, P. T. Spellman, H. Koeppl, G. Stolovitzky, J. Saez-Rodriguez, and S. Mukherjee. Inferring causal molecular networks: Empirical assessment through a community-based effort. Nature methods, 13(4), 2016. URL https://doi.org/10.1038/nmeth.3773. [p366]

J. H. McDonald. Handbook of Biological Statistics, chapter G-test of goodness-of-fit, pages 53-58. Sparkly House Publishing, 3rd edition, 2014. [p366]

W. Patefield. Algorithm AS 159: An efficient method of generating random $\mathrm{R} \times \mathrm{C}$ tables with given row and column totals. Journal of the Royal Statistical Society C, 30(1):91-97, 1981. URL https: //doi.org/10.2307/2346669. [p366]

K. Pearson. X. on the criterion that a given system of deviations from the probable in the case of a correlated system of variables is such that it can be reasonably supposed to have arisen from random sampling. The London, Edinburgh, and Dublin Philosophical Magazine and Journal of Science, 50 (302):157-175, 1900. URL https://doi .org/10.1080/14786440009463897. [p366]

H. A. Simon and N. Rescher. Cause and counterfactual. Philosophy of Science, 33(4):323-340, 1966. URL https://doi.org/10.1086/288105. [p366]

Y. Zhang and M. Song. Deciphering interactions in causal networks without parametric assumptions, 2013. URL https://arxiv.org/abs/1311.2707. [p366, 371]

Y. Zhang, Z. L. Liu, and M. Song. ChiNet uncovers rewired transcription subnetworks in tolerant yeast for advanced biofuels conversion. Nucleic Acids Research, 43(9):4393-4407, 2015. [p366, 371, 372]

Y. Zhang, H. Zhong, R. Sharma, S. Kumar, and J. Song. FunChisq: Chi-Square and Exact Tests for NonParametric Functional Dependencies, 2017. URL https://cran. r-project. org/package=FunChisq. R package version 2.4.0. [p366] 


\section{Ruby Sharma}

Department of Computer Science New Mexico State University

Las Cruces, NM

U.S.A.

ruby49@nmsu. edu

\section{Sajal Kumar}

Department of Computer Science New Mexico State University

Las Cruces, NM

U.S.A.

sajal49@nmsu.edu

Hua Zhong

Department of Computer Science New Mexico State University

Las Cruces, NM

U.S.A.

huazhong@nmsu. edu

Mingzhou Song

Department of Computer Science New Mexico State University

Las Cruces, NM

U.S.A.

ORCiD: 0000-0002-6883-6547

joemsong@cs.nmsu.edu 